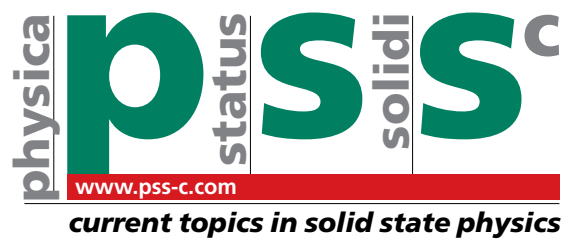

\title{
Surface modification of field emission cathodes made of artificial carbon-based material
}

\author{
Alexander Chepusov ${ }^{*}, 1,2$, Seif Cholakh ${ }^{2}$, Eugene Kislov ${ }^{* *},{ }^{1}$, Alexander Komarsky ${ }^{1,2}$, Vadim Kuznetsov $^{1}$, \\ Sergei Niculin ${ }^{1}$, and Denis Skomorokhov \\ ${ }^{1}$ The Institute of Electrophysics, Ural Division of the Russian Academy of Sciences (IEP UD RAS), 106 Amundsen st., \\ 620016 Ekaterinburg, Russia \\ ${ }^{2}$ Ural Federal University B.N. Yeltsin (UrFU), 19 Mira st., 620002 Ekaterinburg, Russia
}

Received 15 October 2012, revised 10 January 2013, accepted 11 January 2013

Published online 11 March 2013

Keywords field emission cathode, carbon, XPS, Auger spectroscopy, plasmon, hybridization

* Corresponding authors: e-mail chepusov@iep.uran.ru, Phone: +73 432678 766, Fax: +73 432678794

** e-mail keaman@yandex.ru

While the field emission acts at the technical vacuum level conditions $\left(10^{-5} \mathrm{~Pa}\right)$ and electrons emitted from cathode, the ion bombardment exists and continuously modifies the cathode surface. Using X-ray photoelectron spectroscopy (XPS) we studied the electronic structure of thin surface layer of artificial carbon-based materials (ACB) used as field emission cathodes (FEC). The research resulted in the spectra of the samples B-1300, B$1500, \mathrm{~B}-1900$. After spectra processing features analysis shows the change in type of hybridization of the original material. This change is supported by three characteristic spectral features: asymmetry of the core levels spectrum lines change, the satellite structure transformation, Auger-line and modification. So the initial material had $\mathrm{sp}^{2}$ type of hybridization, the spectra show that in thin surface layer a new phase with $\mathrm{sp}^{3}$-hybridization exists and we can conclude formation of nanoclusters with diamond-phase in thin surface layer of cathode.

๑ 2013 WILEY-VCH Verlag GmbH \& Co. KGaA, Weinheim

\section{Introduction}

Creation of stable FEC is the one of the most important challenges in vacuum electronics. It can work at high vacuum conditions with pressure about $10^{-5} \mathrm{~Pa}$, which we call a "technical vacuum". FEC has the next advantages among other electron sources: absence of filament and inertia, high current density, nonlinear current-voltage characteristic. These properties facilitate the application of FEC in different electrical devices: X-ray tubes and others electron-beam sources, light sources, flat displays. The high sensitivity of field emission process to the state of the surface is main difficulty for creating stable field emitter.

Since the authors of famous article [1] about carbonfiber field emission cathode has pointed many of its prospects, such as lifetime, creation simplicity as well as their wide application in commercial electron beam devices, the common interest in carbon materials are rapidly increased.

Field emission properties of graphite cathode are mainly defined by parameters of microprotrusions on its emitting surface [2, 3]. Carbon materials (CM) have several advantages over metal cathodes. They have a high melting point, good vacuum properties, the mastered technology of production, unnecessity to produce a sharp electrode, opportunity to form steady surface configurations with plenty of emitting centers, stability of the chemical composition, they are cheap and available. CM structure is well known. But its surface is affected by ions during the work. And this process is weakly studied.

The residual gases are always present in the volume of any kind of vacuum electron-devices. Under the influence of electrons they are ionized in the space between electrodes during the work and bombard the cathode surface. It also can cause changes in the microstructure of the cathode surface leading to the creation and/or destruction of emission centers. A modification of the cathode emitting surface and therefore the field emission properties are results of the process. 
Processes connected with cathode work are defined by both chemical composition and sample electron structure. Such analysis is provided by X-ray photoelectron spectroscopy (XPS). FEC made of ACM are investigated. Cokecoal tar compositions with different annealing temperatures are considered: B-1300, B-1500 and B-1900. Number in marking means synthesis temperature. These materials are used as binding medium for artificial carbon-based materials producing. Composition properties depend on its synthesis temperature. Higher temperature provides purer substance. Likely, it is the nature of binding medium that defines properties of entire material. Examined cathode sides are named respectively "S1" - active side (worked as FEC) and "S2" - flip side (with original structure and properties).

\section{Experimental}

For specimen atoms excitation aluminum $\mathrm{Al} \mathrm{K \alpha _{1,2 }}$ characteristic X-ray radiation with photons energy $1486.6 \mathrm{eV}$ is used.

Spectra are represented as binding energy $\left(E_{b}\right)$ versus intensity curve. The first one starts from Fermi energy what is equal zero $\left(\mathrm{E}_{\mathrm{F}}=0\right)$.

First of all surface modifications can be observed by means of microscopy. Figure 1 demonstrates atomic field microscopy (AFM) images of B-1300 specimen before (Fig. 1, A) and after field emission (Fig. 1, B). In the first picture we can see a rather smooth relief of the carbon surface with crystallographic plane borders. The second image shows essential surface changes. It gets edged shape with plenty of nanosized peaks. Such morphology changes are accompanied by electron structure transformation.

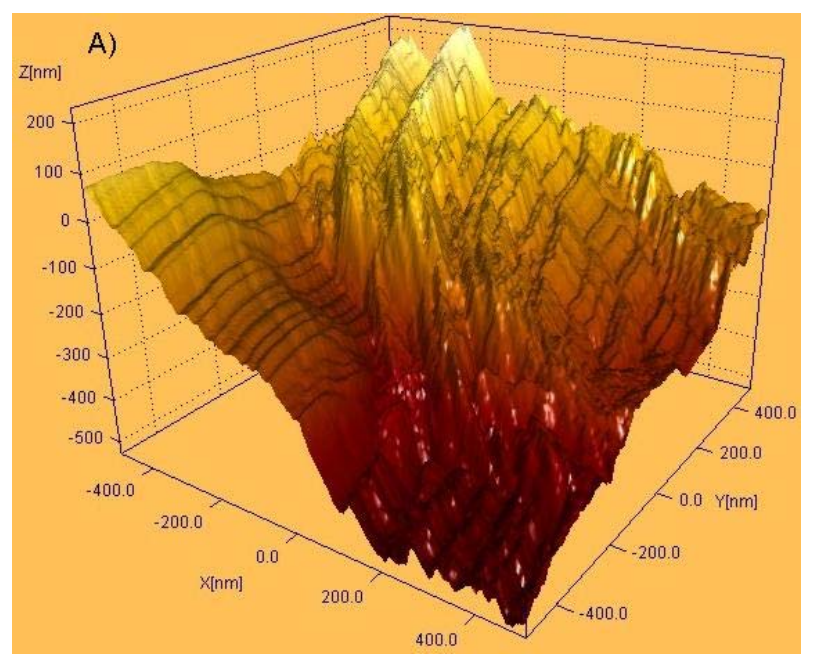

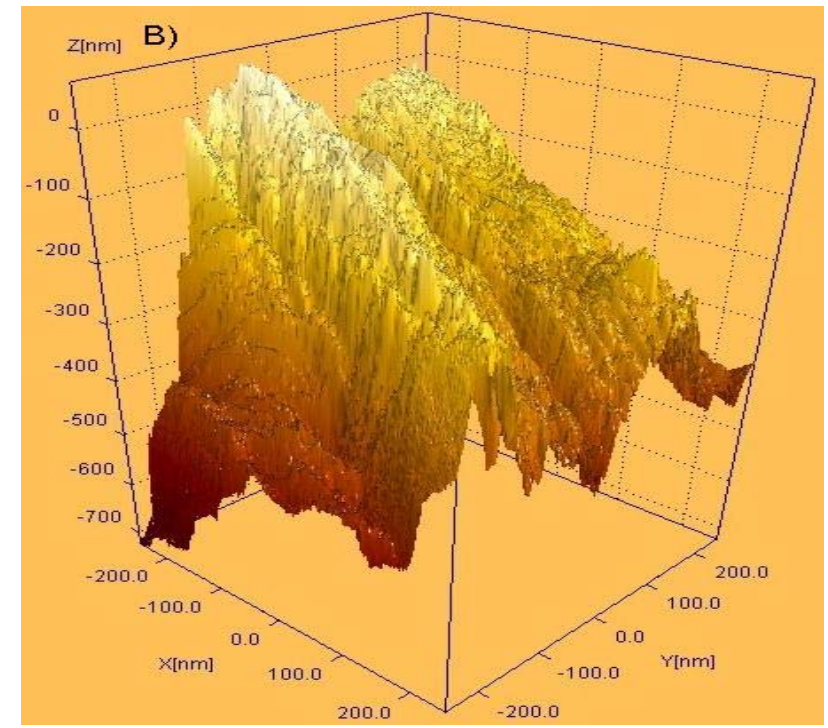

Figure 1 AFM images of B-1300 surface: (A) before field emission; (B) after emission.

2.1 Survey spectra Usually the XPS sample analysis starts from overview spectrum in a wide range of binding energies. Figure 2 shows such spectra for B-1300 sample. It has curves for active (S1) and flip (S2) sides of the specimen. Using this spectrum, it is possible to judge about the chemical composition of the surface and its transformation during the research process. Flip side line contains intense carbon and oxygen spectral lines. They are caused by adsorbed carbon dioxide, water vapor and hydrocarbons. Active side does not have these contaminations because ion bombardment helps to remove them from the surface. However oxygen presents in spectrum and also fluorine, barium, lead, argent and natrium spectral lines appear. The last substances are probably ACM synthesis residual fragments. Spectra analysis shows that specimens B-1500 and B-1900 (samples with higher synthesis temperature) have fewer impurities than B-1300.

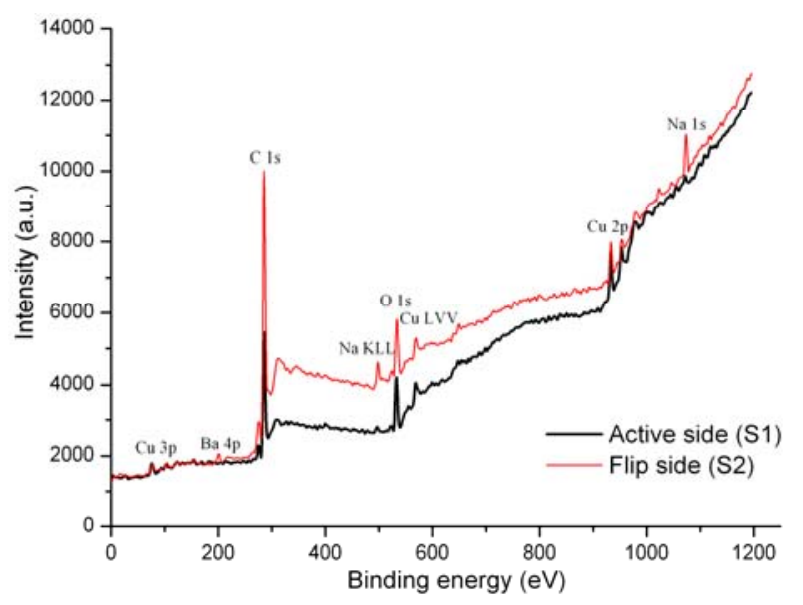

Figure 2 B-1300 survey spectrum: black thick line - active side $\mathrm{S} 1$, red thin line - flip side S2. 
2.2 C 1s-line analysis The next step is carbon core line (C 1s-line) analysis. Carbon allotropes have different carbon line peak position: graphite $-284.5 \mathrm{eV}$, glassy carbon $-285 \mathrm{eV}$, diamond $-285 \mathrm{eV}$ [4]. Often line may be multicomponent. Therefore spectral line can be asymmetric and it has essential asymmetry index. C 1s-line, its features and experimental data have been considered using methods described elsewhere [5], the next interesting fact is established. S1-side has less symmetric C 1s-line, consiquently, greater asymmetry index then S2. Hence, spectrum line is multicomponent. Figure 3 shows B-1900 C 1s-line and its components for both active (Fig. 3 B) and flip side (Fig. 3 A). S2-line for this sample has asymmetry index equal to 0.08 . On the other hand $\mathrm{S} 1$-side has index 0.28 . Thus, spectrum line is multicomponent. Using special method [5] two phase components with energies 284.5 and $285 \mathrm{eV}$ are identified. They correspond to graphite with $\mathrm{sp}^{2}$-hybridization and diamond with $\mathrm{sp}^{3}$-hybridization respectively.
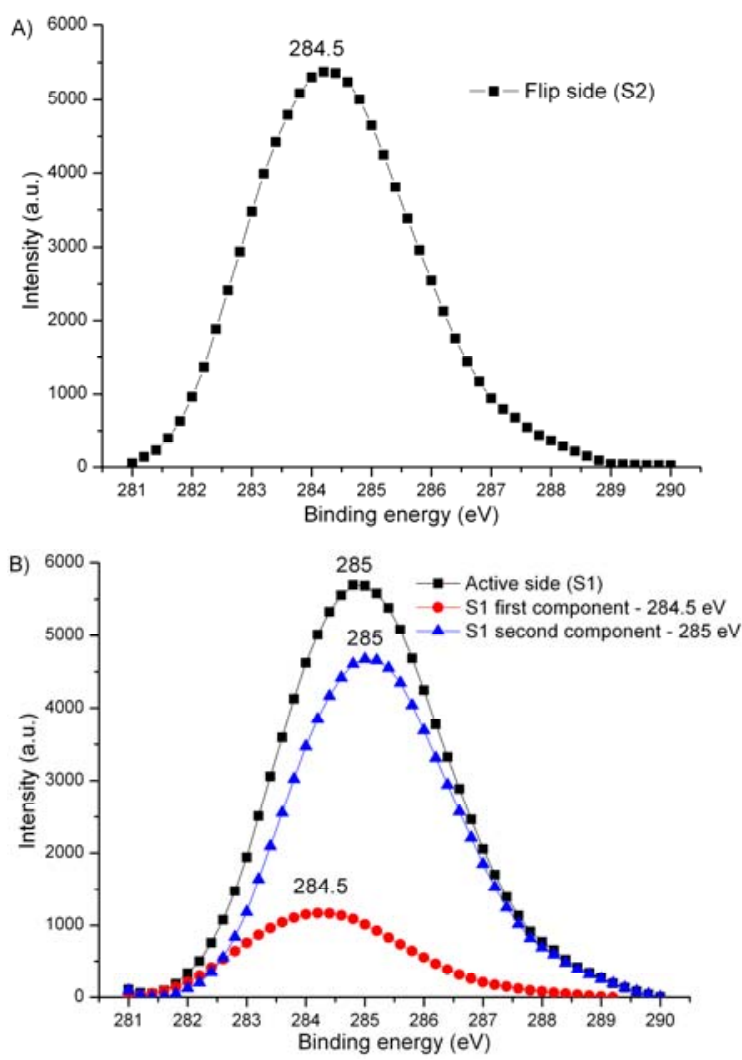

Figure 3 B-1900 C 1s-line: (A) flip side (S2), asymmetry index 0.08 ; (B) active side (S1), asymmetry index 0.28 .

Another marked C 1s feature is plasmon losses spectrum. Energy losses are characteristics of the sample and depend on the chemical composition and material crystal lattice structure. Our specimens demonstrate appreciable wide peaks, what are $20-25 \mathrm{eV}$ away from base carbon line with greater energies. Figure 4 shows processed B-1500 spectrum of plasmon structure. Spectrum transformation is evident. C 1s-line changes its position as well as energy losses peaks. On the S1-spectrum (Fig. 4 B) plasmon lines are well-defined whereas peaks at the flip side spectrum (Fig. 4 A) are smoothed. Quantitative information is described in Table 1.
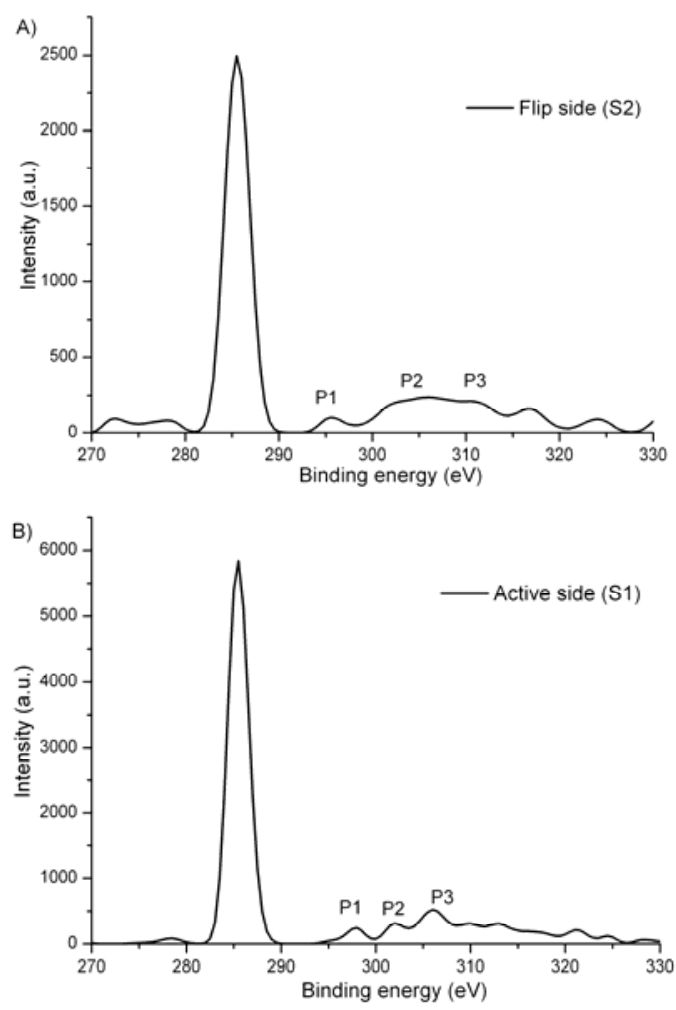

Figure 4 B-1500 plasmon losses spectrum: (A) flip side (S2), (B) active side (S1).

Table 1 Plasmon peaks positions for samples [4].

\begin{tabular}{lllll}
\hline \multirow{2}{*}{ Side } & C 1s-line po- & \multicolumn{3}{c}{ Plasmon distances, eV } \\
& sition, eV & P1 & P2 & P3 \\
\hline B-1300 & & & & \\
Active, S1 & 285,1 & 9 & 14,5 & 22,5 \\
Flip, S2 & 284,5 & 5,5 & 20 & 23 \\
\hline B-1500 & & & & \\
Active, S1 & 284,9 & 11,5 & 16,5 & 21 \\
Flip, S2 & 284,5 & 10 & 20 & 25,5 \\
\hline B-1900 & & & & \\
Active, S1 & 284,9 & 9 & 19 & 26,5 \\
Flip, S2 & 284,6 & 11,5 & 20,5 & 32 \\
\hline
\end{tabular}

A comparison with the data presented in [4] shows that, instead of the initial graphite structure of S2-side (C 1s peak at $284.5 \mathrm{eV}$ ) with $\mathrm{sp}^{2}$-hybridization $\mathrm{S} 1$-spectrum gets diamond features. C 1s position becomes at $285 \mathrm{eV}$ and appropriate intense energy losses peaks appear.

2.3 Auger line analysis Along with the photoelectrons emitted from material in XPS spectra also distribution of Auger electrons presents [6]. Thus, there is a parallel acquisition both XPS and Auger spectra. 
It is known that the Auger electrons are produced as a result of a three-step process with a double ionization of matter atoms. At the recombination stage of the excited electron and then at transferring the difference of energy to another, the valence electrons are most often involved, and therefore the resulting form of the Auger spectrum represent a convolution of the electron levels density distribution and has a complex multicomponent structure. For the same reasons, Auger carbon line is a valence band autoconvolution and also reflects its electronic structure [7]. Whereas the Auger-line is a convolution of the valence band with a core line and appears on the background of intense loss spectrum, the comfortable way to identify their features is to use derivatives. Figure 5 shows derivatives of Auger-line for B-1500.

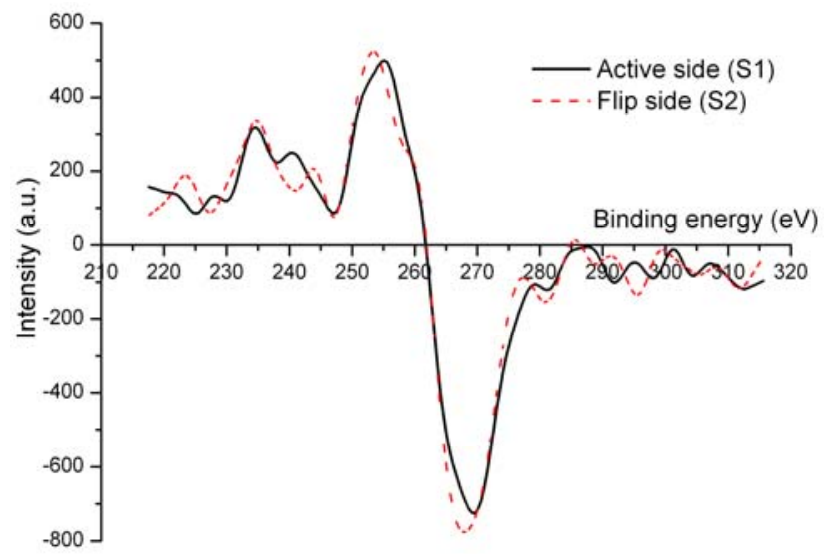

Figure 5 Derivatives of Auger-line for B-1500 sample: black solid line: active side (S1), red dashed line: flip side (S2).

Positive peak form of the Auger-line derivative characterizes the density of states in the carbon valence band. Negative peak position corresponds to bandgap presence. In our case the difference between derivatives negative peaks positions allows supposing hybridization change during field emission [7]. Table 2 shows shifts of the Auger lines for considered samples. The study of the Auger line derivatives shows the formation of the band gap in the surface layer of carbon emitter under ion bombardment with increasing synthesis temperature.

Table 2 Shift of the position Auger-line

\begin{tabular}{ll}
\hline Specimen & Auger-line shift $\Delta \mathrm{E}, \mathrm{eV}$ \\
\hline B-1300 & 0,5 \\
B-1500 & 1,5 \\
B-1900 & 2,5 \\
\hline
\end{tabular}

2.4 Valence band studies The next stage of our work is valence band analysis. In the article [4] valence bands of diamond, glassy carbon and crystalline graphite are investigated by XPS. Figure 6 shows valence bands of these materials.

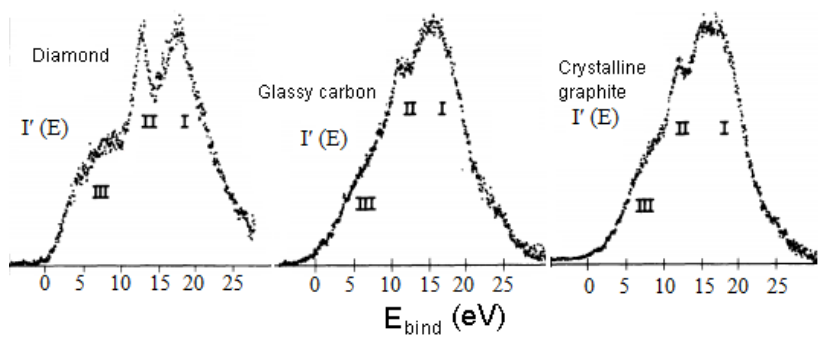

Figure 6 Valence band spectra of diamond, glassy carbon and crystalline graphite.

Each spectrum shows a fairly broad, intense peak located between 16 and $21 \mathrm{eV}$ (peak I), a narrower, less intense peak located at about 10 to $15 \mathrm{eV}$ (peak II), and a very broad and decidedly weaker structure, extending from 10 to $13 \mathrm{eV}$ to the cutoff energy (peak III). There are, however, easily noticeable and significant differences in the spectra. Peak I in diamond is less dominant than its analog in graphite and glassy carbon. In addition, peak III arises sharply in diamond while in graphite it tails off slowly toward low binding energies. Also, in graphite there is a well-defined minimum between peaks I and II which persists even in the microcrystalline sample. This minimum is less pronounced in glassy carbon. Factors accounting for these differences arise from both density-of-states and photoemission cross-section effects [4].

In Fig. 7 the valence band spectra of B-1500 are shown.
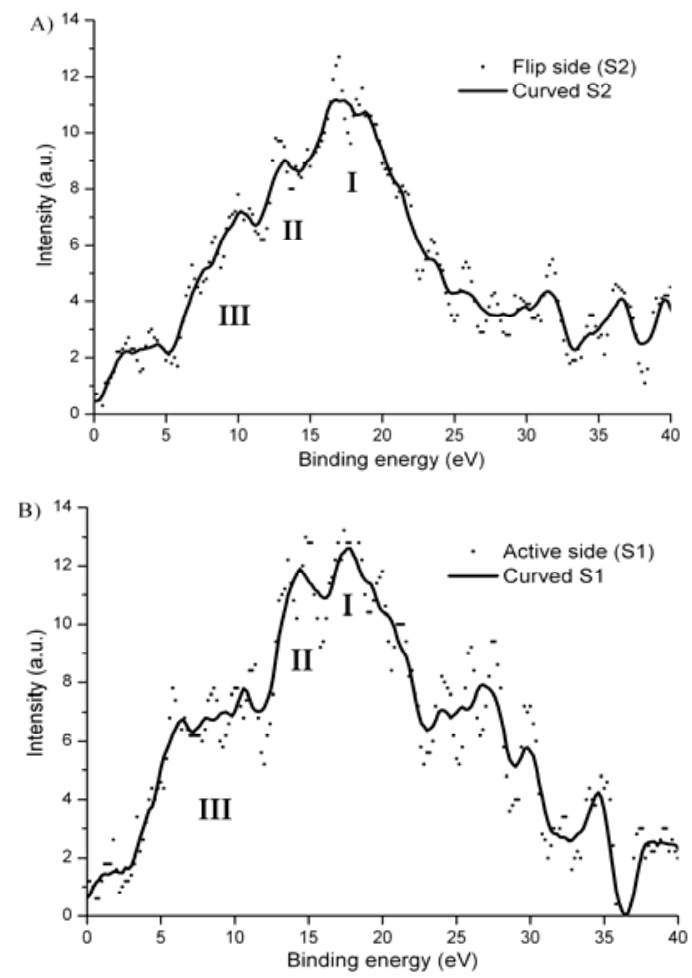

Figure 7 Valence band spectra of B-1500: (A) flip side (S2), (B) active side (S1) after Ar ions exposure. 
S2 side peak I (Fig. 7A) has maximum at $17 \mathrm{eV}$. It is rather wide and much more intensive than peak II (12.5 $\mathrm{eV})$. Zone III is weakly defined. Such band character corresponds to crystalline graphite structure. Active side (S1) spectrum (Fig. 7 B) has intense peaks I and II with almost equal intensities. Peaks positions are 18 and $15 \mathrm{eV}$ respectively. Region III is more clearly distinguished. All these facts prove that there are diamond-like structures on the cathode surface.

Ion bombardment of cathode during its work results in ACM valence band changes and diamond-like clusters generation. Such inclusions have another conductivity type, and according to our model concepts have become polarized, creating additional local accelerating field. This may explain the experimental fact of the field emission start decreasing what is associated with the resulting work function lowering.

3 Conclusions Field-emission cathodes made of ACM have been investigated by XPS. The analysis of the survey spectra has been done, carbon $\mathrm{C} 1 \mathrm{~s}$ line and its satellites have been considered, Auger-electrons line and valence band features of each sample has been investigated. Noted the following: the $\mathrm{C} \mathrm{1s-line} \mathrm{spectra} \mathrm{of} \mathrm{active} \mathrm{sides}$ of the samples include several components with maxima at 284.5 and $285 \mathrm{eV}$. It has clearly expressed satellite lines plasmon loss peaks. The formation of the band gap reflected in the Auger spectra and, finally, the transformation of the valence band are detected. Analysis of the results enables to conclude the formation of inclusions with another type of hybridization and conductivity $\left(\mathrm{sp}^{3}\right.$ instead of $\mathrm{sp}^{2}$, insulator instead of a semimetal, respectively), named according to the type of hybridization diamond-like clusters on the surface of the carbon material. Appearance on the surface with the metal properties of the dielectric inclusions changes the configuration of the electric field, generates additional structure what accelerate the electrons. Thus, the bombardment of the FEC made of ACM by residual gas ions through the generation of diamond-like structures leads to reduction of the resulting work function of specimens.

Acknowledgements The authors are grateful to Gribov I.V. - PhD (physics and mathematics) and senior scientific researcher - for carrying out spectroscopy works, to students of UrFU participated in spectra processing, to engineers of IEP UD RAS for assisting. This work was supported by RFBR under contract number 10-08-00830-a.

\section{References}

[1] F. S. Baker, A. R. Osborn, and J. Williams, Nature 239, 96 (1972).

[2] B. V. Bondarenco, V. I. Macuha, and E. P. Sheshin, Electron. Eng. 10, 44 (1984).

[3] A. L. Suvorov, E. P. Sheshin, and V. V. Protasenco, Tech. Phys. Russ. J. Appl. Phys. 66, 156 (1996).
[4] F. R. McFeely, S. P. Kowalczyk, L. Ley, R. G. Carell, R. A. Pollak, and D. A. Shirley, Phys. Rev. B 12, 5268 (1974).

[5] Y. A. Babanov, I. Y. Kamenski, V. L. Kuznetsov, S. S. Mikhailova, P. V. TItov, and A. L. Filatov, J. Surf. Invest. X-Ray, Synchrotron Neutron Tech. 11, 44 (2006).

[6] D. P. Woodruff and T. A. Delchar, Modern Techniques of Surface Science (Mir Moscow, 1989).

[7] A. A. Galuska, H. H. Madden, and R. E. Allred, Surf. Sci. 32, 253 (1988) 